\title{
Predicting the '97 El Niño event with a global climate model
}

\author{
Josef M. Oberhuber \\ Deutsches Klimarechenzentrum GmbH, Hamburg, Germany \\ E. Roeckner, M. Christoph, M. Esch and M. Latif \\ Max-Planck-Institut für Meteorologie, Hamburg, Germany
}

\begin{abstract}
A simple data assimilation technique has been applied for initializing coupled ocean-atmosphere general circulation models, which is able to generate the threedimensional thermal state of the low-latitude oceans by forcing the model with observed anomalies of sea surface temperature. The scheme has been tested in a multi-year experiment in which the vertical temperature profiles in the equatorial Pacific measured by the TOGA-TAO array have been successfully reproduced for the period '96 to '97. In a further series of eight hindcast experiments initialized between January '96 and September '97, the predictive skill of the model was tested. All experiments starting in ' 97 correctly simulated the evolution of the ' $97 \mathrm{El} \mathrm{Niño,} \mathrm{although}$ the amplitude was slightly underestimated. While the ocean was pre-conditioned to create an El Niño already in '96, the model correctly stayed in the cold (La Niña) phase initially. All experiments initialized in ' 97 forecast a La Niña event for the middle of ' 98.
\end{abstract}

\section{Introduction}

Global climate models are developed for simulating and understanding climate variability, from seasonal to decadal time scales and longer, and also for the prediction of anthropogenic climate change. Although the predictions from different models largely agree in time mean patterns like temperature change, there is less agreement with respect to the El Niño/Southern Oscillation (ENSO) phenomenon [IPCC'96], the most prominent example of interannual variability. A prerequisite for a credible simulation of ENSO change in a warmer climate is a successful simulation of the ENSO statistics under present climate conditions. Although the ENSO simulations of climate models are progressively improving, it is still a common problem in coarse-grid models that the ENSO signal is underestimated due to an inadequate resolution of equatorial Kelvin waves [IPCC'96]. Increasing the resolution certainly has a beneficial effect [Roeckner et al., 1996; Frey et al., 1997; Bacher et al., 1998], and the simulated ENSO events in these models look 'similar' to real ENSO events, however, a unique measure is difficult to obtain.

In this study, a supplementary approach for testing the ENSO physics in climate models is to investigate their forecast skill. For that purpose, a simple data assimilation

\section{Copyright 1998 by the American Geophysical Union.}

Paper number 98GL51782.

0094-8534/98/98GL-51782\$05.00 scheme is proposed which allows climate models to be initialized with observed data without perturbing their background state.

\section{Model Description}

The model used in this study is a state-of-the-art global coupled model [Roeckner et al., 1996] consisting of an isopycnic-coordinate oceanic GCM ('OPYC3') and an atmospheric GCM ('ECHAM4'). The horizontal resolution of the atmosphere and mid- and high latitude ocean model is $2.8^{\circ}$. For a better representation of equatorial wave dynamics, the meridional resolution in the ocean is gradually increased at low latitudes, reaching $0.5^{\circ}$ at the equator. Flux corrections are applied only for the annual mean heat and freshwater fluxes. Since an El Niño event can be regarded as an amplified seasonal cycle, it is important to avoid nonlinear phase locking to a seasonal cycle forced by a seasonal flux correction.

In a 300-year control experiment, the model generated an irregular ENSO cycle which looks realistic with respect to both amplitude and wave propagation [Bacher et al., 1998]. It was also shown that the simulated events are connected with atmospheric anomalies that are similar to the observed [Roeckner et al., 1996]. When applied to an IPCC climate change scenario (IS92a), the model predicts that, in a warmer climate, El Niño's become more frequent, cold events become stronger, the onset of El Niño's is delayed by typically a few months, while the mean zonal temperature gradient in the equatorial Pacific is reduced [Timmermann et al., 1998].

\section{Data assimilation scheme}

Operational ENSO prediction is often based on simplified models consisting of a detailed GCM for the tropical oceans coupled to simpler atmospheric models reproducing the essential characteristics of tropical air-sea interaction [e.g., Zebiac et al., 1987; Barnett et al., 1993]. More recently, weather forecast centers like NCEP and ECMWF have developed global or near-global coupled GCM's for operational seasonal forecasting [Ji et al., 1996; Stockdale et al., 1997]. In these models, the initial state of the atmosphere is taken from the respective operational analysis while global SST's and all available sub-surface thermal data are used to update the ocean state.

A data assimilation scheme (see discussion in Barnett et al., 1993) described below has been applied for use in coupled global climate models, mainly for model validation and 


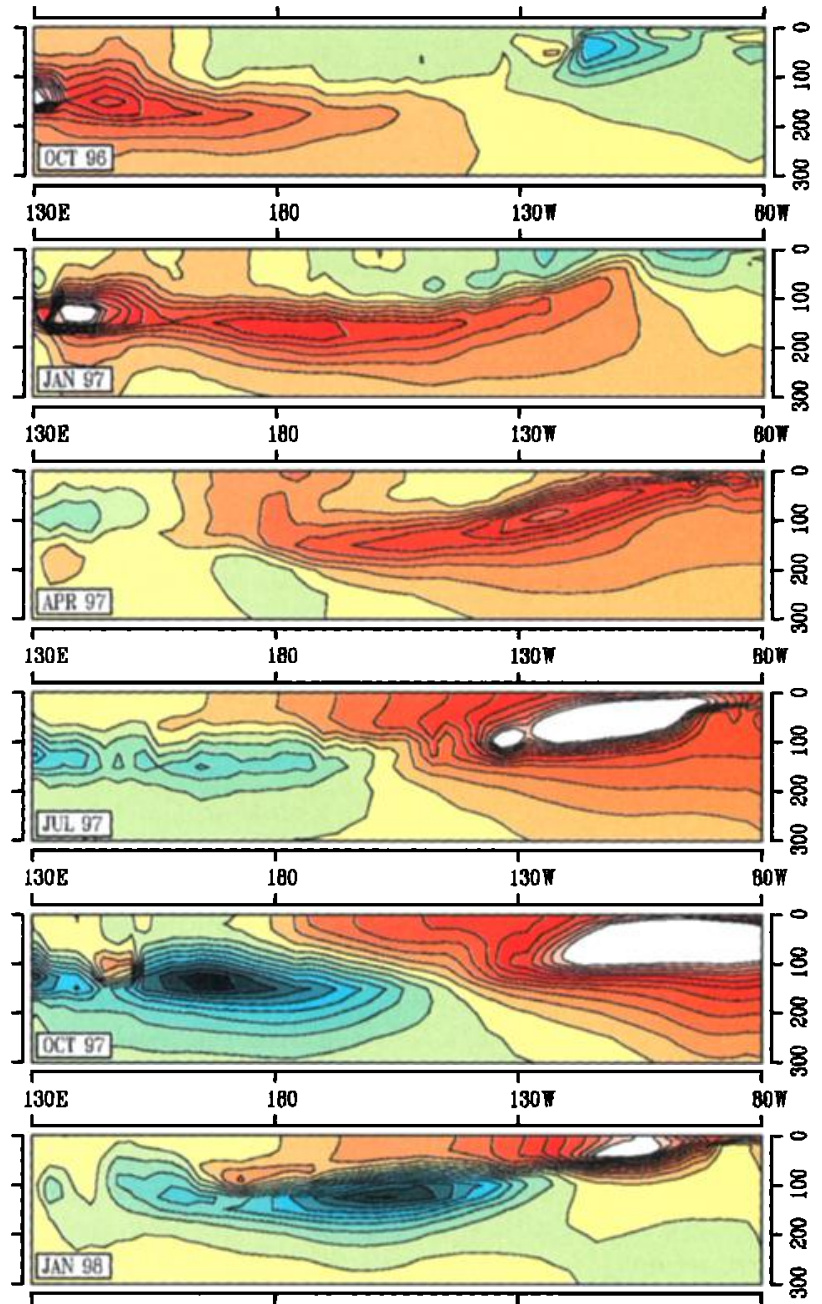

Figure 1. Vertical cross-sections of monthly equatorial temperature anomalies in the data assimilation experiment, and for Jan.'98 in the prediction initialized in Oct.'97. The colour increment is $0.5 \mathrm{~K}$.

intercomparison. The scheme should be (i) easy to apply, (ii) consistent with the model physics, and (iii) create a realistic three-dimensional state of the ocean without initiating a climate drift. A climate drift would be tolerable in the
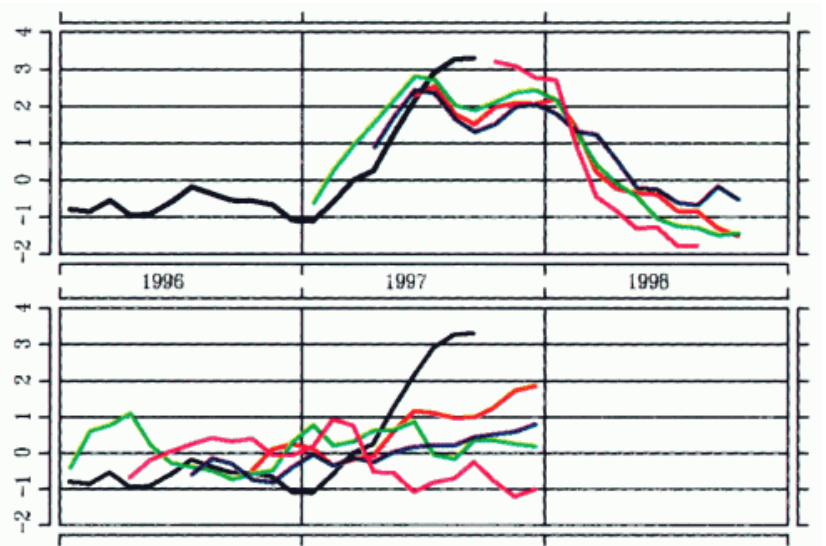

Figure 2. Niño-3 averaged SSTA [K] as observed (black) and for the predictions (coloured) initialized Jan., Apr., Jun. and Oct.'97 (upper panel) and for predictions initialized Jan., Apr., Jul. and Oct.'96 (lower panel).

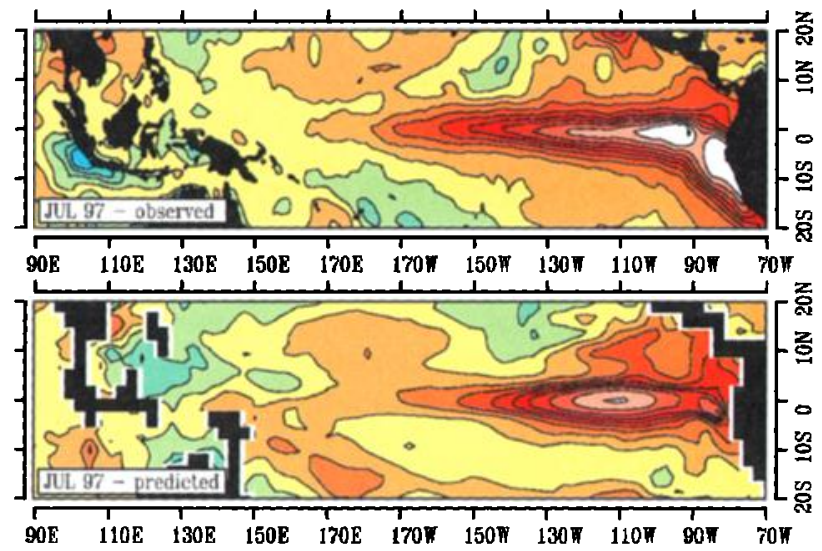

Figure 3. Observed (upper panel) vs. predicted (lower panel) SSTA for Jul.'97. The latter is from the prediction initialized Jan.' 97 . The colour increment is $0.5 \mathrm{~K}$.

extratropics where slow Rossby waves cause an adjustment process with a much longer time scale than that relevant for seasonal forecasting. In the tropics, however, the adjustment is governed by fast Kelvin waves which, at the same time, are one of the key processes governing the evolution of an El Niño event. Therefore, due to the inherent nonlinearity of the dynamics, it would be difficult to separate an El Niño signal from the model's adjustment process.

To initialize the model, a data assimilation experiment was performed in which the model was forced with observed sea surface temperatures, January ' 82 to October ' 97 . However, since this procedure would inevitably create a climate drift, the observed SST record was modified by removing the mean seasonal cycle and replacing it by that obtained from the control run, so that the model is effectively forced with observed SST anomalies. The adjustment, or 'nudging' applied in the assimilation experiment changes with latitude. While it is strong at low latitudes, the coupling to the observations is less tight at middle and high latitudes. Poleward of $60^{\circ}$, no nudging is applied at all, so that ocean and sea ice can develop freely according to the model-generated energy budget. At lower latitudes, the ocean and atmosphere are decoupled with respect to the heat fluxes, while the observed SST anomalies create anomalous forcing of the ocean through atmospheric anomalies, i.e., wind stress and freshwater flux. The wind stress response to the imposed SST anomalies is crucial, because tropical winds create a distribution of oceanic potential energy, or total heat content, that could be the source for Kelvin waves as a response to West Pacific wind anomalies. In fact, the strong low-latitude coupling between the atmosphere and ocean, in particular between SST and wind stress, is the essence of the scheme, and it can only be 'successful' if this coupling is adequately described by the model. Therefore, the assimilation experiment can already be used for model validation, and one may ask to what extent the model would be able to capture the observed thermal state of the ocean, at low latitudes, by forcing the model with observed SST anomalies alone.

As an example, Fig. 1 shows a sequence of cross sections of temperature anomalies along the equatorial Pacific as obtained from the data assimilation experiment. Compared to results from the TOGA-TAO array (McPhaden, 1995; data available through WWW), the model is able to capture the downward displacement of the thermocline in the 

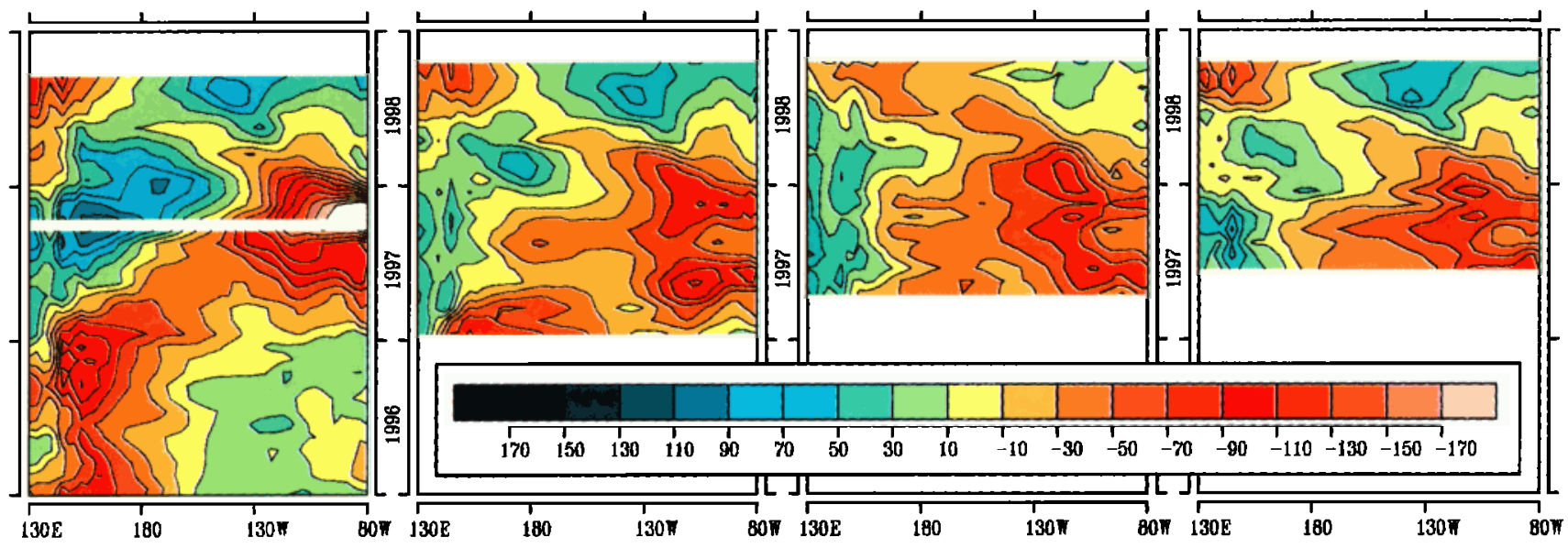

Figure 4. Hovmöller-diagrams of anomalous, top to $350 \mathrm{~m}$ integrated, density averaged between $10 \mathrm{~S}$ and $10 \mathrm{~N}$ in $\mathrm{kg} / \mathrm{m}^{2}$. In the left panel, the lower part refers to the assimilation experiment and the upper part to the prediction initialized Oct.'97. The other panels are predictions initialized Jan., Apr. and Jun.'97.

West Pacific for the pre-El Niño phase (October '96) as well as the eastward propagation, during the El Niño phases, towards the coast of South America. In April '97, the thermal anomaly reaches the surface and the surface warming spreads westward until July. At the same time, an upward displacement of the thermocline, or cold anomaly, starts to develop in the West Pacific, intensifies until October and propagates eastward. This feature is a first indication of a later La Niña event in '98. Because the thermal anomalies at thermocline depth have no connection with those at the sea surface, it can be excluded that processes like subduction of surface water play a role. More likely, the thermal anomalies reflect the impact of atmospheric wind response to imposed SST anomalies.

\section{Results of prediction experiments}

To test the predictive skill of the model, we have performed eight 'hindcast experiments', initialized between January 1, '96 and October 1, '97, and extended over a period of at least one year. The initial states, for both atmosphere and ocean, are taken from the data assimilation experiment explained in section 3. Fig. 2 shows the evolution of Niño-3 SST anomalies as prescribed in the assimilation run according to observations (black) and predicted by the model from various initial states (coloured). All experiments initialized in ' 97 (upper panel) predict an El Niño for ' 97 and a cold (La Niña) event in '98, with remarkably little spread in the predictions. While the warming trend is realistic until about July, the September 'dip' is unrealistic. Although the warming predicted by the model tends to increase again towards the end of the year, it does not reach the observed anomalies of almost $4 K$. As to be expected, the model fails to predict the ' 97 event if initialized in ' 96 (Fig. 2, lower panel) but it correctly avoids generating an El Niño for '96. This is not trivial because there is enough heat stored in the tropical West Pacific due to strong trades in the preceding years (see also Fig. 4). We speculate that different patterns of SST anomalies in the equatorial West Pacific could play a role. In early ' 97 , a warm SST anomaly was observed near the dateline, favoring the occurrence of westerly wind bursts and oceanic Kelvin waves which could trigger the onset of an El Niño event through positive feedbacks between atmosphere and ocean. This positive feedback loop was not triggered in early ' 96 , despite the occurrence of westerly wind bursts in the assimilation experiment (cf., Fig. 5), probably due to the more westward location of the equatorial SST anomaly as compared with ' 97 .
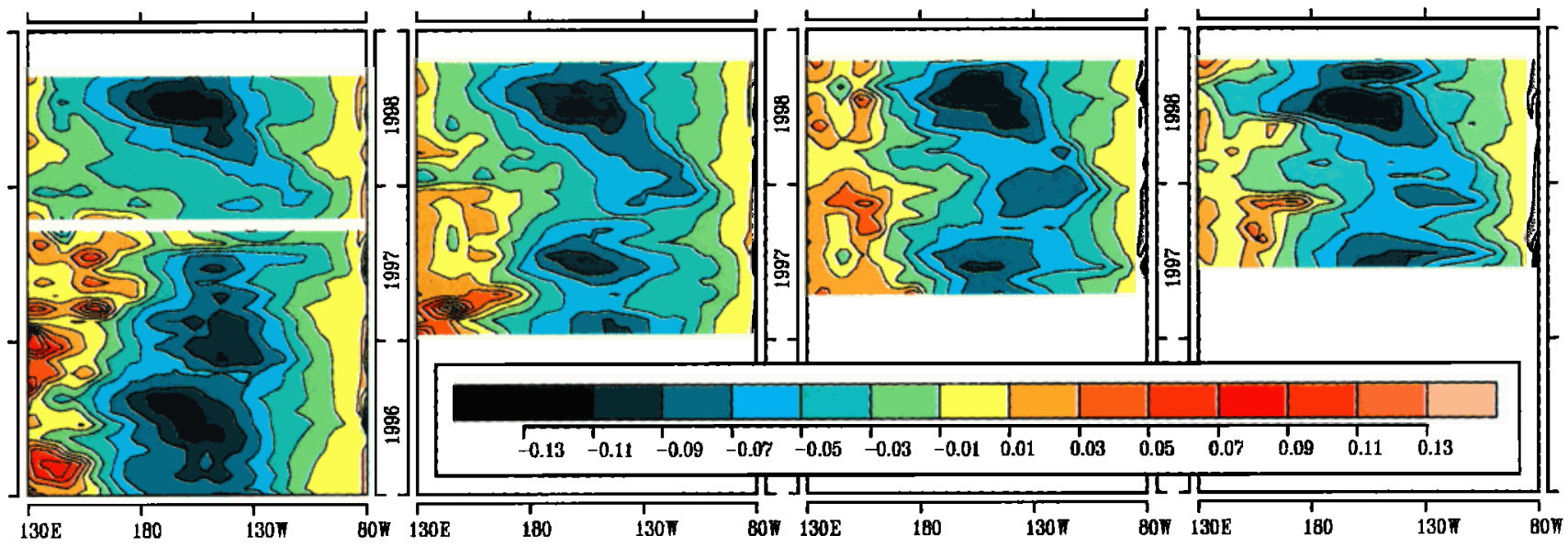

Figure 5. Same as Fig. 4, except for equatorial wind stress in $N / m^{2}$. 
Fig. 3 compares the patterns of observed and predicted SST anomalies for July '97. The experiment was initialized on January 1, '97. Despite the obvious similarities in the patterns, there are a few differences, such as the lack of coastal warming in the model prediction. This has already been noted in the control experiment [Roeckner et al., 1996] and is probably due to the coarse zonal resolution of about $2.8^{\circ}$, which is insufficient to allow poleward propagating coastal Kelvin waves. The equatorial wave guide is better resolved, the meridional resolution of $0.5^{\circ}$ being adequate for equatorial Kelvin waves.

Fig. 4 shows the time evolution of vertically integrated density anomalies along the equatorial belt. At low latitudes this is equivalent to an anomalous heat content. During the assimilation phase, shown in the left part of the upper panel, the positive heat anomaly in the West Pacific remains almost stationary throughout '96. In the beginning of '97, the anomaly starts propagating eastward, initiating the El Niño event in the East Pacific. This can also be seen in the hindcast experiments. At the same time, a negative heat anomaly occurs in the West Pacific which, consistent with most predictions, does not remain stationary but propagates eastward, thus initiating a La Niña event in '98. The corresponding evolution of the wind stress is shown in Fig. 5. In the data assimilation experiment, the West Pacific westerlies in '97 gradually move towards the dateline, while the East Pacific easterlies become weaker during the second half of the year. In '98, which is predicted to be a La Niña year, the westerlies retreat, while the easterlies pick up again during the second half of the year.

\section{Conclusions}

A simple data assimilation scheme has been applied which is able to generate a realistic sub-surface thermal structure in the low-latitude oceans by forcing a coupled model with observed SST anomalies. The method, which relies on readily available data and on the strong low-latitude coupling between atmosphere and ocean, seems particularly useful for the purpose of model validation.

In a series of hindcast experiments, initialized between January ' 96 and October ' 97 , it is shown that our model is able to predict the evolution of the ' $97 \mathrm{El} \mathrm{Niño} \mathrm{with} \mathrm{reason-}$ able skill, although the amplitude is systematically underestimated. It is interesting to note that an El Niño event did not evolve in '96, nor was it predicted, although the ocean was pre-conditioned in early '96 already through a positive heat content anomaly in the West Pacific. All experiments initialized in '97 predict a cold (La Niña) event for '98. This is supported by both the TOGA-TAO array data and the assimilation experiment which show a cold subsurface anomaly propagating eastward.

Although hindcast experiments have been made so far only for a single event, the results look sufficiently promising to warrant further testing of the assimilation/hindcast scheme in coupled models. In particular, the scheme may be useful for projects like the 'Coupled Model Intercomparison Project' (CMIP), because predictive skill could be readily assessed in many models without placing too much burden on the individual modeling groups. However, due to internal atmospheric variability the statistical significance of predictions needs to be optimized by use of a multi-integration ensemble approach similar to that employed in operational weather forecast.

Acknowledgments. This work was sponsered by the German government under its program 'Klimavariabilität und Signalanalyse'. The model integrations were performed at the Deutsches Klimarechenzentrum GmbH (DKRZ). We thank L. Bengtsson and A. Miller for careful reading of the manuscript and for useful comments.

\section{References}

Bacher, A., J.M. Oberhuber and E. Roeckner, ENSO dynamics and seasonal cycle in the tropical Pacific as simulated by the ECHAM4/OPYC3 coupled general circulation model, Clim. Dyn., in press, 1998.

Barnett, T.P., M. Latif, N.E. Graham, M. Flügel, S. Pazan and W. White, ENSO and ENSO-related predictability. Part I: Prediction of equatorial Pacific sea surface temperature with a hybrid coupled ocean-atmosphere model, J. Climate, 6, 15451566, 1993.

Frey, H., M. Latif and T. Stockdale, The coupled GCM ECHO-2. Part I: The Tropical Pacific, Mon. Weather Rev., 125, 703-720, 1997.

Ji, M., A. Leetmaa and V.E. Kousky, Coupled model predictions of ENSO during the 1980s and 1990s at the National Centers for Environmental Prediction, J. Climate, 9, 3105-3120, 1996.

IPCC, Climate Change 1995, The Science of Climate Change, 572 pp., Cambridge University Press, Cambridge, 1996.

McPhaden, M.J., The tropical atmosphere ocean (TAO) array is completed, Bull. Am. Meteorol. Soc., 76, No. 5, 1995.

Roeckner, E., J.M. Oberhuber, A. Bacher, M. Christoph and I. Kirchner, ENSO variability and atmospheric response in a global coupled atmosphere-ocean GCM, Clim. Dyn., 12, 737$754,1996$.

Stockdale, T.N., Coupled ocean-atmosphere forecasts in the presence of climate drift, Mon. Weather Rev., 125, 809-818, 1997.

Timmermann, A., J.M. Oberhuber, A. Bacher, M. Esch, M. Latif and E. Roeckner, ENSO response to greenhouse warming, Max-Planck-Institut für Meteorologie, Hamburg, Rep. No. 251, 13 pp., 1998.

Zebiak, S.E. and M.A. Cane, A model El Niño-Southern Oscillation, Mon. Weather Rev., 115, 2262-2278, 1987.

J.M. Oberhuber, Model Development and Application Group, German Climate Computing Centre, Bundesstr. 55, 20146 Hamburg, Germany. (email: oberhuber@dkrz.de)

M. Christoph, M. Esch, M. Latif and E. Roeckner, Max-PlanckInstitut für Meteorologie, Bundestr. 55, 20146 Hamburg, Germany. (e-mail: christoph@dkrz.de; esch@dkrz.de, latif@dkrz.de, roeckner@dkrz.de )

(Received February 18, 1998; accepted April 24, 1998.) 